\title{
Magnetoresistive effects in Co/Pd multilayers on self-assembled nanoparticles (invited)
}

\author{
Judith Kimling née Moser, ${ }^{1,2, a)}$ Vojko Kunej, ${ }^{1}$ Hans-Fridtjof Pernau, ${ }^{1}$ Elke Scheer, ${ }^{1}$ and \\ Manfred Albrecht ${ }^{1,3}$ \\ ${ }^{1}$ Department of Physics, University of Konstanz, D-78457 Konstanz, Germany \\ ${ }^{2}$ Institute of Applied Physics and Microstructure Research Center Hamburg, University of Hamburg, D- \\ 20355 Hamburg, Germany \\ ${ }^{3}$ Institute of Physics, Chemnitz University of Technology, D-09107 Chemnitz, Germany
}

(Presented 21 January 2010; received 31 October 2009; accepted 5 January 2010; published online 5 May 2010)

\begin{abstract}
The deposition of $\mathrm{Co} / \mathrm{Pd}$ multilayers onto self-assembled spherical particles provides a system with unique magnetic properties. The magnetic caps have high perpendicular magnetic anisotropy, are single-domain, and strongly exchange decoupled, but in electrical contact with each other, thus enabling magnetotransport measurements. By applying an external magnetic field, the caps can be switched individually. We systematically studied the magnetoresistance on a two-dimensional cap array consisting of $\mathrm{Co} / \mathrm{Pd}$ multilayers deposited on particles with a diameter of $200 \mathrm{~nm}$. In the vicinity of the coercive field, a hysteretic resistance peak occurs. It can be explained with the random magnetization configuration of the magnetic caps leading to an increased spin-dependent scattering of the conduction electrons. The underlying mechanism might be comparable to the one causing giant magnetoresistance in granular alloys. For temperatures above $77 \mathrm{~K}$, additional resistivity contributions with high saturation fields are observed, which are tentatively explained by the decreasing size of magnetically ordered parts of the caps with increasing temperature, resulting finally in superparamagnetic behavior in the contact area between neighboring caps. () 2010 American Institute of Physics. [doi:10.1063/1.3350909]
\end{abstract}

\section{INTRODUCTION}

Magnetic nanostructures are of considerable interest for both fundamental research and industrial applications. Besides exhibiting novel magnetic effects, the potential of magnetic nanostructures is given by the possibility to engineer their specific properties, which strongly depend on the geometry and the material composition. ${ }^{1,2}$ Multilayer and nanocomposite structures as well as the confinement into two dimensions or one dimension can lead to exciting physical phenomena such as giant magnetoresistance (GMR), tunnel magnetoresistance (TMR), exchange bias, and spin-torque induced dynamics or reversal. ${ }^{3-5}$ There are worldwide major research efforts in the field of magnetic nanostructures. Current and potential applications include information storage, sensor technology, biomedical applications including drug delivery, and spintronic devices. ${ }^{5-7}$

Conventional methods for the creation of magnetic nanostructures are top-down and bottom-up techniques combining optical or electron-beam lithography, chemical or physical etching, and material deposition. In recent years, a variety of new concepts for the fabrication of well-ordered nanostructures with defined magnetic properties were presented. They range from the self-organization of magnetic particles $^{8,9}$ over the self-organization of magnetic entities embedded in hosts, e.g., block copolymers, ${ }^{10,11}$ and the deposition of magnetic films on substrates prepatterned by self-

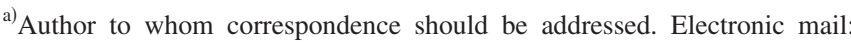
jmoser@physnet.uni-hamburg.de.
}

organized material ${ }^{12}$ to the employment of self-assembled structures as a mask. ${ }^{10}$ In this regard, another innovative approach to create nanostructure arrays is the deposition of $\mathrm{Co} / \mathrm{Pt}$ and $\mathrm{Co} / \mathrm{Pd}$ multilayers onto monolayers of selfassembled nanoparticles. ${ }^{13-15}$ The system provides unique curvature-induced magnetic properties combined with the advantages of patterning magnetic structures by means of self-assembly over large areas. The effective anisotropy of $\mathrm{Co} / \mathrm{Pd}$ multilayers in general can be tailored by adjusting the deposition parameters and individual layer thicknesses. For Co layers up to $1.6 \mathrm{~nm}$, the interface anisotropy dominates over volume anisotropy and leads to a magnetic easy axis perpendicular to the interfaces. ${ }^{16}$ The effect can be enhanced by the magnetostriction of interfacial Co-Pd alloys. ${ }^{17}$ On the other hand, the interface anisotropy is reduced by an increasing interface roughness. ${ }^{18}$ The deposition of $\mathrm{Co} / \mathrm{Pd}$ multilayers onto spherical particles provides an array of magnetic cap structures. For Co layers below $0.8 \mathrm{~nm}$, the curvature of the nanoparticles leads to a radial spread of the anisotropy axis pointing perpendicular to the surface of the particles. Apart from the orientation of the anisotropy axis, its strength varies according to the layer thickness. ${ }^{13}$ The thickness of the deposited layers decreases from the top of each particle towards the sides. If the thickness of the Co layers falls below one monolayer, the system forms a dilute Co-Pd alloy. It is assumed that comparable Co-Pd alloys are not ferromagnetic at room temperature, ${ }^{19,20}$ which is suggested to result in a strong magnetic exchange decoupling of the caps. The decoupling is further supported by the small contact areas between neighboring particles. Up to a particle diameter of 


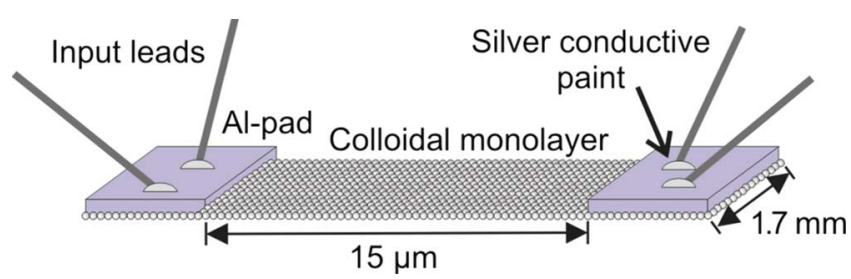

FIG. 1. (Color online) Sketch of the sample layout. Two input leads were bonded on each of the aluminum pads.

about $300 \mathrm{~nm}$ the $\mathrm{Co} / \mathrm{Pd}$-multilayer caps are quasi-single domain. The magnetic properties of the system such as magnetic anisotropy and coercive field can be tailored by adjusting the deposition parameters and by simply using particles of appropriate size. ${ }^{15}$

So far, the magnetic properties of this novel kind of nanostructures were systematically studied by superconducting quantum interference device (SQUID) and magnetooptical Kerr-effect magnetometry. ${ }^{13-15}$ In this article, we report on MR measurements on a two-dimensional (2D) cap array. Transport measurements are a powerful tool for the characterization of magnetic nanostructures because electrical current is highly sensitive to the magnetic moments of a sample and, unlike other techniques, the MR signal does not scale with the size of the probed area. Apart from displaying the magnetization reversal of the magnetic caps where most of the magnetic material is deposited, MR measurements can provide access to the magnetic properties of the dilute $\mathrm{Co}-\mathrm{Pd}$ alloy in the contact regions between neighboring caps.

\section{SAMPLE PREPARATION AND EXPERIMENTAL METHOD}

For the fabrication of 2D particle arrays, negatively charged polystyrene spheres with a diameter of $200 \mathrm{~nm}$ were used. Monolayers of these particles were prepared in a selfassembly process as described by Micheletto et al. ${ }^{21}$ As substrate we employed a standard silicon wafer with a silicon dioxide surface layer. Since the quality of the 2D crystal growth depends on the particle mobility, the substrate was treated with oxygen plasma to remove organic impurities and to increase the hydrophilicity. The original colloidal suspension was diluted to about $1 \%$ by volume, applied to the substrate, and left to dry in a closed chamber that was tilted about $5^{\circ}$ to cause a directed crystal growth. ${ }^{22}$ The particle monolayer was then covered with 30 repetitions of $\mathrm{Co}(0.4 \mathrm{~nm}) / \mathrm{Pd}(0.8 \mathrm{~nm})$ bilayers on a seed layer of $\mathrm{Cr}(1 \mathrm{~nm}) / \mathrm{Pd}(5 \mathrm{~nm})$. The deposition was done by magnetron sputtering from concentric ring cathodes at an argon pressure of $11 \mu$ bar. The growth rates were $1.4 \mathrm{~nm} / \mathrm{s}$ for Co and 7.3 $\mathrm{nm} / \mathrm{s}$ for Pd. To prevent oxidation, the multilayers were covered with an additional $0.8 \mathrm{~nm}$ thick Pd layer.

For the magnetic characterization of the sample, the domain configuration was imaged by means of magnetic force microscopy (MFM) at room temperature. Temperaturedependent magnetization curves were recorded by means of SQUID magnetometry. MR measurements were performed in a pseudo four-terminal setup, as illustrated in Fig. 1. To ensure a densely packed assembly of particles and to avoid short circuiting of the caps on the colloidal monolayer by the

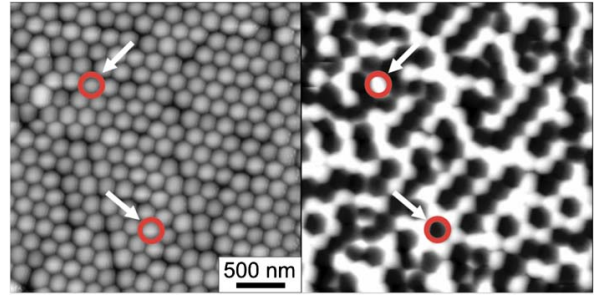

FIG. 2. (Color online) Atomic force micrograph revealing topography (left) and corresponding MFM image of $[\mathrm{Co}(0.4 \mathrm{~nm}) / \mathrm{Pd}(0.8 \mathrm{~nm})] \times 30$ multilayer film deposited on a monolayer of $200 \mathrm{~nm}$ polystyrene particles. The arrows and circles mark the same particles in both images.

flat $\mathrm{Co} / \mathrm{Pd}$ multilayer film deposited on the substrate, the sample was reduced to a $1.7 \mathrm{~mm}$ wide piece. Two aluminum pads with a thickness of $500 \mathrm{~nm}$ and a distance of $15 \mu \mathrm{m}$ were defined using a thin wire as evaporation mask. As input leads two wires were bonded on each pad. Transport measurements were done with an ac resistance bridge with a maximum applied current of $10 \mu \mathrm{A}$. The signal was read out by a multimeter. The sample was cooled down in a cryostat with variable temperature insert. Measurements were done in helium atmosphere between 77 and $260 \mathrm{~K}$. A magnetic field up to $10 \mathrm{~T}$ was applied perpendicular to the sample plane and the current direction.

\section{RESULTS AND DISCUSSION}

Figure 2 shows a magnetic force micrograph of the prepared sample. The individual caps appear in a well-defined black or white contrast confirming that they are single domain. As the demagnetized state of the sample is driven by magnetostatic interactions, a mazelike overall domain pattern can be observed. Figure 3(a) presents a magnetization curve measured by SQUID magnetometry at $77 \mathrm{~K}$ together with a typical resistance-versus-field curve recorded at 77.5 K. Both curves exhibit hysteretic behavior. The magnetization curve indicates an out-of-plane anisotropy of the $\mathrm{Co} / \mathrm{Pd}$-multilayer caps with a coercive field $H_{C}$ of $90 \mathrm{mT}$. The broad switching field distribution (SFD) mainly originates from the nonuniform particle-size distribution. However, at small separations between nanostructures, the dipole-dipole interaction becomes important and influences the magnetization reversal in the array. ${ }^{23-26}$ This explains why the remanence of the normalized magnetization curves is not one. At higher temperatures, the general shape of the magnetization curves remains the same, only the coercive field is reduced to $75 \mathrm{mT}$ at $150 \mathrm{~K}$ and to $64 \mathrm{mT}$ at $300 \mathrm{~K}$.

The MR ratio is defined as $\left[R\left(\mu_{0} H\right)-R_{0}\right] / R_{0}$, where $R\left(\mu_{0} H\right)$ is the measured resistance of the sample at an applied magnetic field $\mu_{0} H$ and $R_{0}=R(1 T)$. The main influence of the external magnetic field on the resistance occurs in the vicinity of the coercive field $H_{C}$ at about $100 \mathrm{mT}$. The slight offset between $H_{C}$ and the peak position of the MR curves can be attributed to different reasons. First, the recording methods are different. While for the SQUID measurement a certain field is applied and the magnetization is measured after the relaxation of the system, the resistance is recorded on the fly with a sweep rate of $0.2 \mathrm{~T} / \mathrm{min}$ and an integration time of 3 s. An additional possibly temperature-dependent 

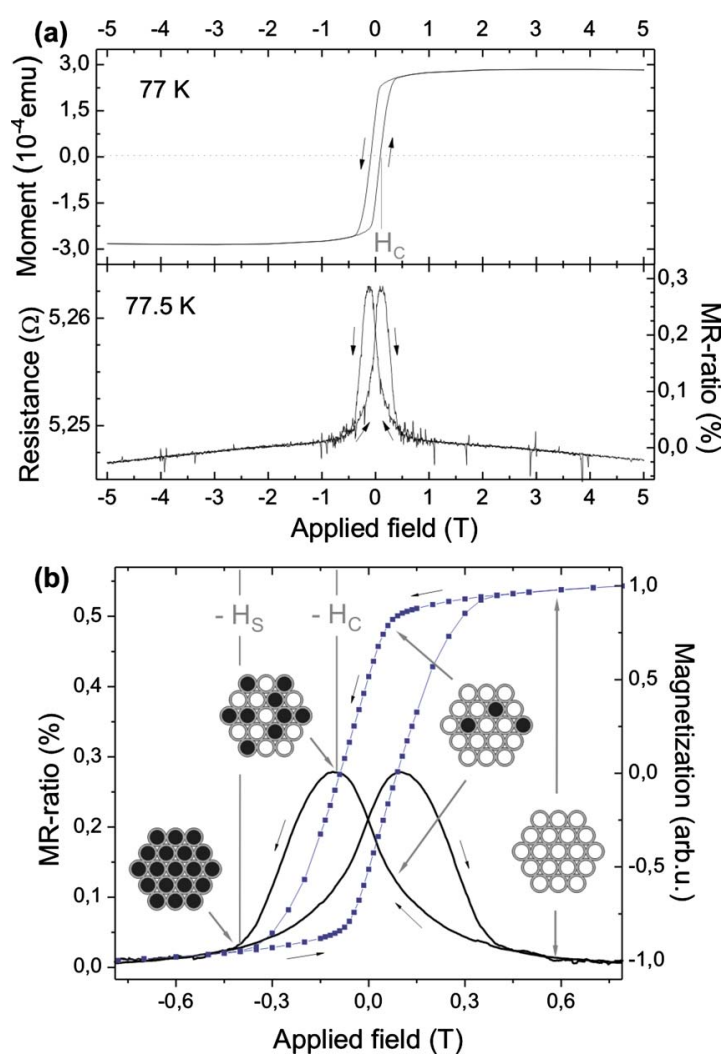

FIG. 3. (Color online) (a) Magnetization and MR of single domain $\mathrm{Co} / \mathrm{Pd}$ multilayer caps on $200 \mathrm{~nm}$ particles. (b) Scale up of the magnetization curve together with a ten-curves average of the MR where the field was swept between $\pm 1 \mathrm{~T}$. The insets show the magnetization configurations corresponding to the respective applied fields. The light (dark) circles represent the single domain caps with the magnetization directions up (down) along the easy axis, which is perpendicular to the sample plane.

contribution to the offset originates from the fact that a SQUID measures the total moment of a sample, while the resistivity is only sensitive to the current-carrying paths.

For higher fields, the sample shows negative MR. This is usually associated with suppressed spin-disorder scattering in a magnetic field. ${ }^{27}$ For the interpretation of the hysteretic resistivity contribution, we propose a model which is illustrated in Fig. 3(b). The system consists of single-domain magnetic caps which are in metallic contact. The magnetization direction of a cap can point up or down perpendicular to the sample plane, as indicated by the light and dark shaded circles. The gray areas are metallic regions where due to the thickness variation across the particle surface the deposited Co-Pd stack loses its multilayered structure, as illustrated in Fig. 4. We will get back to this later. At $+1 \mathrm{~T}$ the sample magnetization is saturated and all cap moments are aligned in parallel. When the field is decreased, some caps start to switch. The magnetization is reduced and the resistivity increases. At $-H_{C}$, where the net sample magnetization is zero, one half of the cap magnetizations points up and the other half points down. If we assume the caps to be magnetically decoupled, their magnetizations are statistically distributed in this demagnetized state. ${ }^{14}$ From the view of spin-dependent transport, this state corresponds to the most disordered or antiferromagnetically aligned state, where the resistivity is the highest. When the reverse field is further increased the
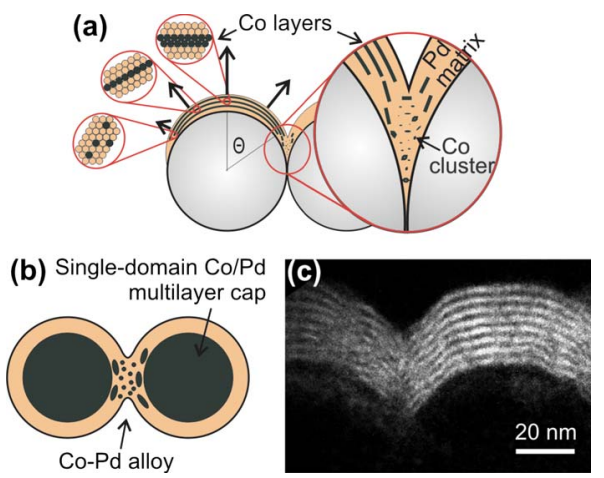

FIG. 4. (Color online) Schemes of (a) the cross section and (b) the top view of $\mathrm{Co} / \mathrm{Pd}$ multilayers deposited on spherical particles. Due to the curvature of the spheres the multilayer structure smears out toward the sides. At the intersection the system forms a granular Co-Pd alloy. The arrows indicate the anisotropy axis. (c) Transmission electron micrograph of a thicker $\mathrm{Co} / \mathrm{Pd}$ multilayer on $50 \mathrm{~nm}$ silicon oxide particles: $[\operatorname{Co}(1.8 \mathrm{~nm}) / \operatorname{Pd}(1.8 \mathrm{~nm})] \times 8$.

magnetic moments of the caps become aligned along the field direction. Spin-dependent scattering of the conduction electrons is reduced and the resistivity decreases. At the saturation field $-H_{S}$ all cap moments are aligned in parallel and the resistivity is lowest. The curvature change of the resistivity curve at $-H_{S}$ supports this model.

Figures 4(a) and 4(b) are schematic side and top views of two neighboring caps. Two different regions can be distinguished: the single domain cap with a well defined $\mathrm{Co} / \mathrm{Pd}$ multilayer structure and the intersection where the multilayers smear out and a granular alloy is formed. To support this model, a reference system of thicker multilayers was prepared for imaging by means of transmission electron microscopy. ${ }^{15}$ The analysis of the energy-filtered transmission electron micrograph shown in Fig. 4(c) reveals a clear multilayer contrast at the central part of the caps while at the intersections the $\mathrm{Co} / \mathrm{Pd}$ interfaces are no longer visible and a granular structure arises.

The present system is to some extent comparable to an alloy consisting of ferromagnetic particles embedded in a metal matrix. GMR is not only observed in layered structures, but also in magnetically inhomogeneous media with single domain ferromagnetic entities, ${ }^{28,29}$ so we might deal with a GMR-like effect. At the intersection between neighboring caps, a magnetization configuration similar to the one in a domain-wall is possible. Furthermore the curvatureinduced radial anisotropy distribution and the complexity of possible current paths lead to contributions from anisotropic MR, which are hard to evaluate. ${ }^{30}$ For this purpose, sophisticated micromagnetic simulations are required, which lie beyond the scope of this work.

Together with the seed and capping layer, the Pd makes up $70 \%$ of the deposited material. Even if the $\mathrm{Pd}$ is partially polarized, there is a significant shunting of the sensing current through the nonmagnetic layers. This explains why the observed MR ratio amounts to $0.3 \%$ only.

In order to confirm that the MR peaks really occur due to the switching of the magnetic caps, minor loops were recorded. Minor loops can be employed to distinguish between reversible and irreversible transitions of the sample configuration. A transition is considered irreversible if a domain 


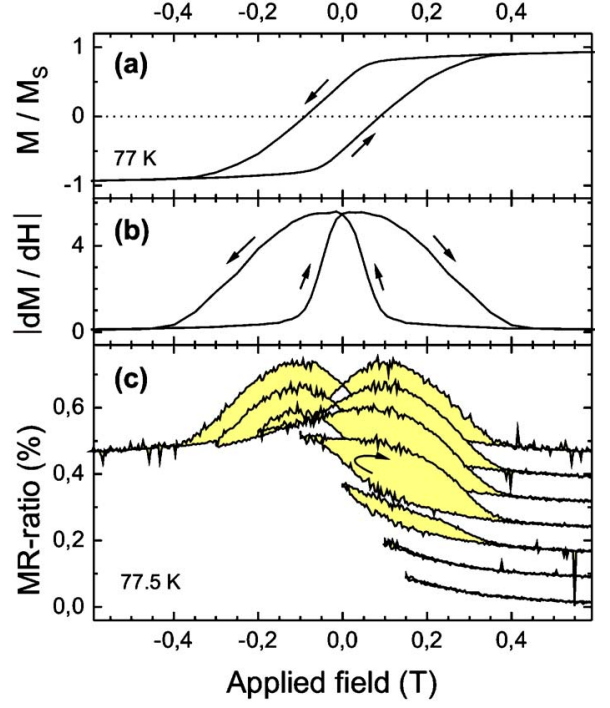

FIG. 5. (Color online) (a) Normalized hysteresis loop measured by SQUID at $77 \mathrm{~K}$. (b) For a better insight into the magnetization reversal process, the derivative of the sample magnetization $d M / d H$ is shown. (c) Minor loops recorded at $77.5 \mathrm{~K}$. Apart from the lowest one the curves are shifted for clarity.

nucleates $^{31}$ or a cap switches. MR minor loops recorded at $77 \mathrm{~K}$ are shown in Fig. 5 together with a SQUID measurement of the sample magnetization and its derivative. For the minor loops, the sample was first saturated at $1 \mathrm{~T}$. The field was then cycled between $1 \mathrm{~T}$ and subsequently decreasing values. Up to a field of $0.1 \mathrm{~T}$, the minor loops are closed. A small increase of the resistivity is observed while the magnetization curve is constant. This points towards a reversible field-dependent resistivity contribution from regions with small magnetic moments. They are not detected by the SQUID since they are hidden by the much higher moments of the magnetic caps (see below). At a field of about $0.1 \mathrm{~T}$, the shape of the minor loops changes. Comparison with the magnetization curves shows that this is the field at which the magnetization of the caps starts to reverse. If the field is decreased below $0.1 \mathrm{~T}$, the loops open up and close again when the saturation field of about $0.4 \mathrm{~T}$ as indicated by the SQUID curve is reached. Even though the mechanisms leading to the SQUID and the MR signal are different, this is a strong indication for the nonreversible contribution to the MR being caused by the switching of the caps. Since the mechanisms causing the reversible part of the curves are unknown, the curves can only be discussed qualitatively. After subtracting the reversible MR contribution, the shape of the minor loops reflects the SFD of the magnetic caps. ${ }^{32}$

Figure 6 shows minor loops recorded at $150 \mathrm{~K}$. The shape of the loops that can be associated with the switching of the caps is comparable to the one observed at $77 \mathrm{~K}$. An interesting feature becoming more obvious in this illustration is, that the MR ratio can be increased in a demagnetizationlike process: if approaching from $-1 \mathrm{~T}$, the field direction is reversed between $+H_{C}$ and $+0.2 \mathrm{~T}$, the peak at $-H_{C}$ is remarkably higher. Consequently, the state at which the magnetization passes through zero is not the most disordered state in terms of spin-dependent scattering. The resistivity being higher in the demagnetized state than at $H_{C}$ is often observed in GMR systems. ${ }^{29,33}$

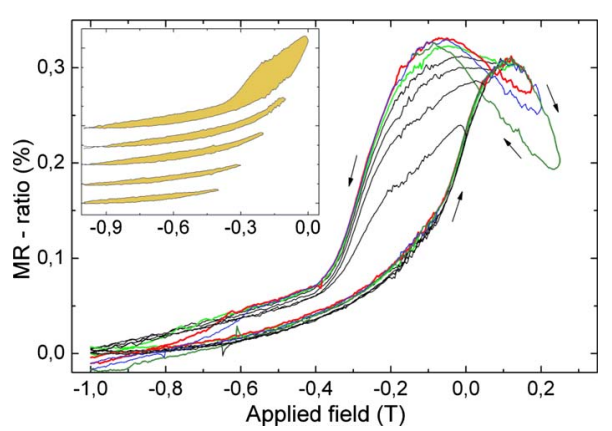

FIG. 6. (Color online) Minor loops recorded at $150 \mathrm{~K}$. The small cycles are shown in the inset with the curves being shifted for clarity.

In contrast to the minor loops recorded at $77 \mathrm{~K}$, the curves at $150 \mathrm{~K}$ are not closed even for small cycles. This hints at additional resistivity contributions occurring with increasing temperature. Figure 7 shows MR traces in fields up to $10 \mathrm{~T}$ recorded at temperatures between 77 and $260 \mathrm{~K}$. The MR ratios of the curves were calculated with $R_{0}=R(0 T)$ and the curves are shifted for clarity. Some cycles are not closed due to thermal drift. The contribution of the magnetic caps in the vicinity of the coercive field $H_{C}$ remains the same for all temperatures. With increasing temperature additional hysteretic resistivity contributions occur, one of which saturates around $4 \mathrm{~T}$. The peak position of these contributions increases with temperature and lies between 0.5 and $0.85 \mathrm{~T}$. The saturation field is also increasing with temperature. For $260 \mathrm{~K}$, no saturation is reached even at $10 \mathrm{~T}$. We assume that the described effects originate from the intersections between neighboring magnetic caps. Since most of the voltage drops there, the transport measurement is
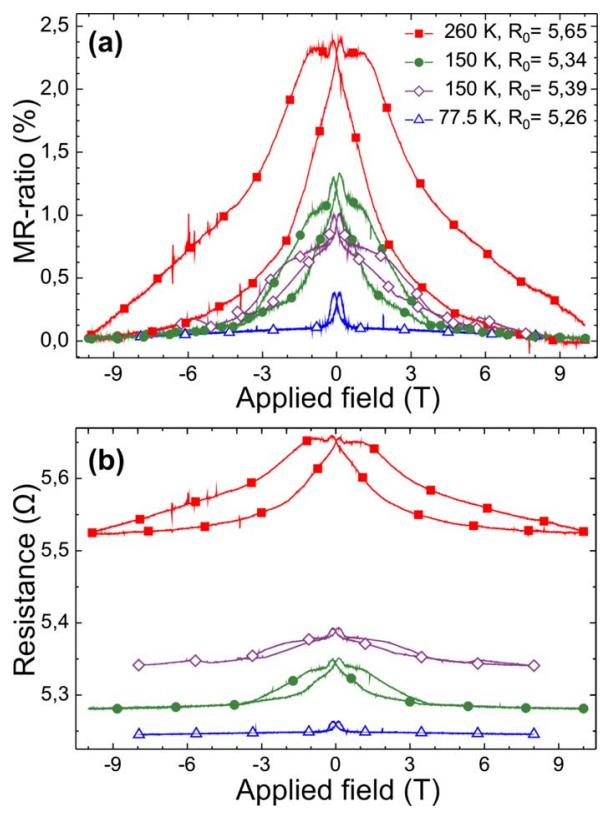

FIG. 7. (Color online) MR sweeps with a maximum applied field between 8 and $10 \mathrm{~T}$ recorded at different temperatures. (a) For better comparison curves are shifted to an MR ratio of zero at the highest applied field. (b) Corresponding absolute resistances of the nonshifted curves. The resistivity is very likely a more accurate hint to the real sample temperature than the displayed temperature because the heat transfer between sensor and sample was poor. 
highly sensitive to these regions. Note that the magnetic properties of the Co-Pd alloy in these areas cannot be detected by SQUID measurements, thus they can only be inferred from the MR curves. The origin of the individual contributions cannot be clarified unambiguously from our data. Generally speaking, one could expect such an alloy to exhibit GMR, ${ }^{28,29}$ but the observed temperature dependence raises questions. Due to the deposition of multilayer stacks, it is possible that oblate Co clusters with peculiar magnetic properties may have formed, which reveal an activated behavior with high saturation field. The increasing saturation field with increasing temperature hints at superparamagnetic behavior. ${ }^{34}$ With an increase in temperature, the Co particles might become progressively superparamagnetic. Superparamagnetic clusters can act as so-called "loose spins." They are known to mediate coupling between ferromagnetic entities. ${ }^{35}$ This would explain both the fact that the additional resistivity contribution only occurs at high temperatures and the hysteretic behavior.

Another possible mechanism which could give rise to an increasing MR ratio with temperature as well as to the unusual shape of the MR traces is the following: We model our samples by two regions one of which is given by the bulk of the individual caps with their clear multilayer structure. The other important areas are the disordered contact regions (compare Fig. 4). Since the majority of the electrical potential drops at the disordered regions, i.e., the intersections, these parts of the sample give an important contribution to the resistance and thus to the MR. The size of the magnetically ordered parts of the caps is expected to decrease with increasing temperature. Simultaneously the disordered parts increase and so does the absolute value of the resistance and the area in which the conduction electrons are spin scattered. Since no detailed calculations of the MR of disordered magnetic systems exist, we can only speculate that this fact might give rise to enhanced MR ratios. With the same model, the larger switching fields at higher temperature can be explained because smaller magnetic grains need higher external fields for alignment.

Large variations of MR curves including sign changes of the GMR are typical observations in atomic contacts ${ }^{36-38}$ in qualitative agreement with calculations of the geometry dependence of the spin polarization, which becomes important at the atomic level. ${ }^{39-42}$ It has been shown that the magnetic properties of all relevant length and size scales of a sample are reflected in the MR traces. Although the contacts in our present system most likely have larger cross-sections than single atoms, a similar mechanism might be active.

\section{CONCLUSION}

A 2D array of $\mathrm{Co} / \mathrm{Pd}$ multilayer caps on self-assembled particles was characterized by MFM, SQUID magnetometry, and magnetotransport measurements. The system investigated provides single-domain magnetic caps with perpendicular magnetic anisotropy. In the vicinity of the coercive field, a hysteretic resistance peak appears. We propose a model, in which the magnetic caps constitute single-domain magnetic particles with a perpendicular anisotropy axis em- bedded in a metal host. The mechanism responsible for the observed magnetoresistive effect might be similar to the one causing the GMR effect in granular alloys. However, further possible origins such as anisotropic MR due to a domainwall-like magnetization configuration between neighboring caps have to be taken into consideration as well. At temperatures above $77 \mathrm{~K}$, additional hysteretic resistivity contributions with saturation fields up to $10 \mathrm{~T}$ occurred, the origin of which remains unclear to date.

Using a lateral magnetic array such as the $\mathrm{Co} / \mathrm{Pd}$ multilayer caps on self-assembled nanospheres as a model system for an alloy consisting of monodisperse particles the sizes of which are known and can be varied in a defined manner, might facilitate further experiments exploring fundamental questions of spin-dependent transport.

\section{ACKNOWLEDGMENTS}

The authors would like to thank O. Hellwig, J. D. Risner-Jamtgaard, and D. Yaney (Hitachi GST) for TEM imaging and $\mathrm{H}$. Rohrmann (OC Oerlikon Balzers AG) for preparing $\mathrm{Co} / \mathrm{Pd}$ multilayers. We also thank S. Fischer, U. Rüdiger, M. Kläui, and D. Makarov for providing equipment and fruitful discussions, as well as M. Suty, M. Keil, and M. Hagner for technical assistance at the University of Konstanz. The DFG is gratefully acknowledged for financial support via the Emmy-Noether-Program. The project was partially funded by the Baden-Wuerttemberg Stiftung within the research network Functional Nanostructures and by BMBF and DFG in the framework of a German-Israeli Project (DIP).

${ }^{1}$ J. I. Martín, J. Nogués, K. Liu, J. L. Vicent, and I. K. Schuller, J. Magn. Magn. Mater. 256, 449 (2003).

${ }^{2}$ F. J. Himpsel, J. E. Ortega, G. J. Mankey, and R. F. Willis, Adv. Phys. 47, 511 (1998).

${ }^{3}$ A. Barthélémy, V. Cros, J. L. Duvail, A. Fert, R. Morel, F. Parent, F. Petroff, and L. B. Steren, Nanostruct. Mater. 6, 217 (1995).

${ }^{4}$ J. Nogués, J. Sort, V. Langlais, V. Skumryev, S. Suriñach, J. S. Muñoz, and M. D. Baró, Phys. Rep. 422, 65 (2005).

${ }^{5}$ I. Žutić, J. Fabian, and S. Das Sarma, Rev. Mod. Phys. 76, 323 (2004).

${ }^{6}$ D. N. Lambeth, E. M. T. Velu, G. H. Bellesis, L. L. Lee, and D. E. Laughlin, J. Appl. Phys. 79, 4496 (1996).

J. Dobson, Drug Dev. Res. 67, 55 (2006).

${ }^{8}$ C. Petit, A. Taleb, and M.-P. Pileni, Adv. Mater. 10, 259 (1998).

${ }^{9}$ S. Sun, C. B. Murray, D. Weller, L. Folks, and A. Moser, Science 287, 1989 (2000).

${ }^{10}$ M. Lazzari and M. A. López-Quintela, Adv. Mater. 15, 1583 (2003).

${ }^{11}$ S. Park, D. H. Lee, J. Xu, B. Kim, S. W. Hong, U. Jeong, T. Xu, and T. Russell, Science 20, 1030 (2009).

${ }^{12}$ M. Grobis, E. Dobisz, O. Hellwig, M. E. Schabes, G. Zeltzer, T. Hauet, and T. R. Albrecht, Appl. Phys. Lett. 96, 052509 (2010).

${ }^{13}$ M. Albrecht, G. Hu, I. L. Guhr, T. C. Ulbrich, J. Boneberg, P. Leiderer, and G. Schatz, Nature Mater. 4, 203 (2005).

${ }^{14}$ T. C. Ulbrich, D. Makarov, G. Hu, I. L. Guhr, D. Suess, T. Schrefl, and M. Albrecht, Phys. Rev. Lett. 96, 077202 (2006).

${ }^{15}$ T. C. Ulbrich, C. Bran, D. Makarov, O. Hellwig, J. D. Risner-Jamtgaard, D. Yaney, H. Rohrmann, V. Neu, and M. Albrecht, Phys. Rev. B 81, 054421 (2010).

${ }^{16}$ F. J. A. den Broeder, W. Hoving, and P. J. H. Bloemen, J. Magn. Magn. Mater. 93, 562 (1991).

${ }^{17}$ J. Hong, S. Sankar, A. Berkowitz, and W. Egelhoff, Jr., J. Magn. Magn. Mater. 285, 359 (2005).

${ }^{18}$ P. F. Carcia, A. D. Meinhaldt, and A. Suna, Appl. Phys. Lett. 47, 178 (1985).

${ }^{19}$ D. M. S. Bagguley, W. A. Crossley, and J. Liesegang, Proc. Phys. Soc. 90, 1047 (1967). 
${ }^{20}$ J. R. Childress, J. L. Duvail, S. Jasmin, A. Barthelemy, A. Fert, A. Schuhl, O. Durand, and P. Galtier, J. Appl. Phys. 75, 6412 (1994).

${ }^{21}$ R. Micheletto, H. Fukuda, and M. Ohtsu, Langmuir 11, 3333 (1995).

${ }^{22}$ F. Burmeister, C. Schäfle, T. Matthes, M. Böhmisch, J. Boneberg, and P. Leiderer, Langmuir 13, 2983 (1997).

${ }^{23}$ P. Krone, D. Makarov, T. Schrefl, and M. Albrecht, J. Appl. Phys. 106, 103913 (2009)

${ }^{24}$ L. C. Sampaio, R. Hyndman, F. S. de Menezes, J. P. Jamet, P. Meyer, J. Gierak, C. Chappert, V. Mathet, and J. Ferre, Phys. Rev. B 64, 184440 (2001).

${ }^{25}$ K. Nielsch, R. B. Wehrspohn, J. Barthel, J. Kirschner, U. Gösele, S. F Fischer, and H. Kronmüller, Appl. Phys. Lett. 79, 1360 (2001)

${ }^{26}$ V. Raposo, J. M. Garcia, J. M. González, and M. Vázquez, J. Magn. Magn. Mater. 222, 227 (2000)

${ }^{27}$ I. Campbell and A. Fert, Ferromagnetic Materials 3, 747 (1982).

${ }^{28}$ A. E. Berkowitz, J. R. Mitchell, M. J. Carey, A. P. Young, S. Zhang, F. E. Spada, F. T. Parker, A. Hutten, and G. Thomas, Phys. Rev. Lett. 68, 3745 (1992).

${ }^{29}$ J. Q. Xiao, J. S. Jiang, and C. L. Chien, Phys. Rev. Lett. 68, 3749 (1992). ${ }^{30}$ M. Bolte, M. Steiner, C. Pels, M. Barthelmess, J. Kruse, U. Merkt, G. Meier, M. Holz, and D. Pfannkuche, Phys. Rev. B 72, 224436 (2005).
${ }^{31}$ J. E. Davies, O. Hellwig, E. E. Fullerton, G. Denbeaux, J. B. Kortright, and K. Liu, Phys. Rev. B 70, 224434 (2004).

${ }^{32}$ A. Berger, B. Lengsfield, and Y. Ikeda, J. Appl. Phys. 99, 08 E705 (2006).

${ }^{33}$ W. P. Pratt, S.-F. Lee, J. M. Slaughter, R. Loloee, P. A. Schroeder, and J. Bass, Phys. Rev. Lett. 66, 3060 (1991).

${ }^{34}$ B. J. Hickey, M. A. Howson, S. O. Musa, and N. Wiser, Phys. Rev. B 51, 667 (1995)

${ }^{35}$ J. C. Slonczewski, J. Appl. Phys. 73, 5957 (1993).

${ }^{36}$ M. Viret, S. Berger, M. Gabureac, F. Ott, D. Olligs, I. Petej, J. F. Gregg, C. Fermon, G. Francinet, and G. Le Goff, Phys. Rev. B 66, 220401 (2002).

${ }^{37}$ M. Viret, M. Gabureac, F. Ott, C. Fermon, C. Barreteau, G. Autès, and R. Guirardo-Lopez, Eur. Phys. J. B 51, 1 (2006).

${ }^{38}$ S. Egle, C. Bacca, H.-F. Pernau, M. Huefner, D. Hinzke, U. Nowak, and E. Scheer, Phys. Rev. B (in press).

${ }^{39}$ G. Autès, C. Barreteau, D. Spanjaard, and M.-C. Desjonquères, J. Phys.: Condens. Matter 18, 6785 (2006).

${ }^{40}$ M. Häfner, J. K. Viljas, D. Frustaglia, F. Pauly, M. Dreher, P. Nielaba, and J. C. Cuevas, Phys. Rev. B 77, 104409 (2008).

${ }^{41}$ G. Autès, C. Barreteau, D. Spanjaard, and M.-C. Desjonquères, Phys. Rev. B 77, 155437 (2008)

${ }^{42}$ M. Häfner, J. K. Viljas, and J. C. Cuevas, Phys. Rev. B 79, 140410 (2009). 